\title{
TIEMPOS DE RECOMPOSICIÓN DE LA DIVERSIDAD ARBÓREA A LO LARGO DE LA SUCESIÓN VEGETAL EN LOS BOSQUES DEL VALLE DE CHANCHAMAYO / JUNÍN / PERÚ
}

\author{
RECOMPOSITION TIMINGS OF TREE DIVERSITY AND PLANT \\ SUCCESION IN FORESTS OF THE CHANCHAMAYO VALLEY / JUNIN \\ / PERU
}

\author{
Flavio Quintero Cardozo ${ }^{1}$, Belvi Margui Cáceres Rodríguez ${ }^{2}$, Carlos Augusto Reynel \\ Rodríguez ${ }^{1,3}$, Robin Fernandez-Hilario ${ }^{1,4}$, Akira Armando Wong Sato ${ }^{1,4}$, Jorge Mario \\ Chávez Salas ${ }^{1}$ y Sonia Cesarina Palacios Ramos ${ }^{1}$
}

\section{Resumen}

Un tema de interés actual en la ciencia forestal concierne a la regeneración de los bosques y áreas degradadas. Dentro de esta temática, un aspecto de importancia se refiere a los tiempos que diferentes forestas demoran en recomponerse de modo natural, recuperando sus niveles originales de diversidad y otras características que son claves para retomar su funcionamiento como ecosistemas. El presente trabajo se centra en el marco de los bosques húmedos premontanos de la selva central de Perú, en el valle de Chanchamayo, Junín, entre 1000 - $1500 \mathrm{msnm}$. Un total de 19 Transectos Gentry de $2 \times 500 \mathrm{~m}$, que incluyen todas las plantas leñosas $\geq 2.5 \mathrm{~cm}$ de Diámetro a la Altura del Pecho fueron establecidos en áreas de bosques maduros, y bosques de diferentes edades posteriores a talado sin quema. Se consideró 5 edades del bosque, $5-10,20,30,40$ y $\geq 50$ años. Se comparó y analizó la diversidad-alfa y la composición de la flora arbórea bajo cada una de estas condiciones. Se observó que, a partir de 40 años de edad, el índice de diversidad Alfa de Fisher se hace bastante similar al que caracteriza a bosques maduros; a partir de 30 años de edad, la composición taxonómica por especies alcanzó una similitud de 69 - 73\%, como las que ocurren en bosques maduros. Las familias, géneros y especies botánicas características en cada una de las edades fueron comparadas, precisando que a medida que se incrementa la edad del bosque, existen menos especies compartidas con un alto número de individuos. Los bosques tempranos, de hasta 20 años de edad, son caracterizados por la presencia de Piperaceae; a partir de los 30 años lo son por la familia Moraceae.

Palabras clave: bosques premontanos, bosques secundarios, dinámica forestal, diversidad arbórea, Transectos Gentry.

\begin{abstract}
A current topic in forestry concerns the regeneration of forests and degraded lands. An important aspect in this respect refers to the timing that different forests take to recover naturally, recomposing their original levels of diversity and other parameters key to their functioning as ecosystems. The present work focuses on the above mentioned, in premontane humid forests of central Peru, in the valley of Chanchamayo, Department of Junín, between $1000-1500$ masl. A total of 19 Gentry's Transects of $2 \times 500 \mathrm{~m}$, including all woody plants $\geq 2.5 \mathrm{~cm}$ Diameter at Breast Height, were performed in areas of mature forests, and forests of different ages subsequent to clearing without burning. Alpha-diversity and composition of the tree flora under these conditions were compared and analyzed. From 40 years of age on, the value of Fisher's Alfa diversity index becomes quite similar to the one of mature forests; from 30 years onwards, the taxonomic composition of species under different ages reaches a similarity of $69-73 \%$ with those in mature forests. Families, genera and species were compared, and it was found out that forests up to 20 years are characterized by the presence of Piperaceae; from 30 years on, by the Moraceae family.

Key words: premontane forests, secondary forests, forest dynamic, tree diversity, Gentry Transects.
\end{abstract}

\section{Introducción}

En los últimos años se han desarrollado trabajos orientados a comprender mejor la composición florística de la selva central de Perú, y cómo ésta caracteriza a los diferentes tipos de bosque existentes en el ámbito (Vásquez et al., 2005; Monteagudo \& Huamán, 2010; Marcelo \& Reynel, 2014). Actualmente la iniciativa de establecimiento de parcelas 
permanentes bajo metodologías estándar en áreas de bosques tropicales ha abierto la puerta a remediciones sucesivas en el tiempo, facilitando una visión de la dinámica de los bosques. Equipos de investigación como la Red Amazónica de Inventarios Forestales (RAINFOR) se encuentran actualmente liderando trabajos que cubren áreas geográficamente amplias permitiendo conocer mejor la composición de la flora, los patrones de distribución de taxones y la dinámica forestal.

En el Departamento de Junín la mayor parte de estudios florísticos, se han realizado en la Provincia de Chanchamayo (Antón \& Reynel, 2004; Cáceres \& Reynel, 2010; Aguilar \& Reynel, 2011; Palacios \& Reynel, 2011; Buttgenbach et al., 2012; Reynel, 2012), pero a pesar de estos esfuerzos, éste sigue siendo uno de los departamentos mayoritariamente amazónicos con menores niveles de colecciones y conocimiento botánico (Honorio \& Reynel, 2003). A esto se debe agregar que, en Chanchamayo y alrededores, más del $80 \%$ del territorio ha sido deforestado (Reynel \& León, 1989), escenario generado por la conversión de uso de tierras de protección y uso forestal a agricultura o ganadería.

Siguiendo las metodologías de RAINFOR (Phillips et al., 2009) se ha podido generar información relevante sobre la composición de los diferentes tipos de bosque existentes en el valle de Chanchamayo, a través de la instalación de Parcelas Permanentes de monitoreo que incluyen el marcado de árboles con más de $10 \mathrm{~cm}$ de Diámetro a la Altura del Pecho (DAP) (Antón \& Reynel, 2004; Aguilar \& Reynel, 2011; Buttgenbach et al., 2012; Giacomotti \& Reynel, 2018). Estos estudios nos han permitido comprender mejor la estructura y composición florística de los bosques montanos y premontanos en función del estado sucesional en el que se encuentran.

Hasta la fecha, los estudios realizados en los bosques húmedos premontanos del valle de Chanchamayo revelan que bosques pioneros establecidos a partir de una quema se caracterizan por presentar en su etapa inicial especies leñosas como Vernonanthura patens (Kunth) H. Rob. (Asteraceae) y Acalypha spp. (Euphorbiaceae) entre las más importantes; luego de 25 años, la composición tiende a ser dominada por especies como Piper aduncum L. y Allophylus foribundus (Poepp.) Radlk. (Sapindaceae) (Echía et al., 2019). Por otro lado, los bosques secundarios tardíos presentan especies como Trophis caucana (Pittier) C.C. Berg (Moraceae), Cupania cinerea Poepp. (Sapindaceae), Inga edulis Mart. (Fabaceae) y Mauria heterophylla Kunth (Anacardiaceae) (Almeyda, 2004). En los bosques primarios, las especies más representativas con DAP < $40 \mathrm{~cm}$ son Batocarpus costaricensis Standl. \& L.O. Williams (Moraceae), Inga cinnamomea Spruce ex Benth. (Fabaceae) y Trophis caucana (Pittier) C.C. Berg (Moraceae), y con DAP $>40 \mathrm{~cm}$, Clarisia racemosa Ruiz \& Pav. (Moraceae) y Pseudolmedia laevis (Ruiz \& Pav.) J.F. Macbr. (Moraceae) (Reynel \& Antón, 2004).

Actualmente, en el valle de Chanchamayo, la gran mayoría de bosques primarios remanentes, o aquellos donde la intervención antrópica ha sido mínima, se encuentran localizados en áreas con pendientes pronunciadas y, por ende, de difícil acceso; mientras que los bosques secundarios, provenientes de áreas anteriormente dedicadas al cultivo del café y cítricos, presentan una gran variedad de edades y se encuentran cubriendo gran parte del valle. Si bien los estudios anteriormente mencionados nos están permitiendo comprender la composición y estructura de estos bosques, aún quedan muchos vacíos de conocimiento por estudiar. Prueba de ello es que, en los últimos 30 años, otros trabajos de enfoque taxonómico han generado el descubrimiento de nuevas especies arbóreas endémicas para la selva central (Pipoly III, 1996; Arroyo, 2014; Berg \& Ulloa Ulloa, 2014). Asimismo, muy pocos estudios se han enfocado en conocer la diversidad de plantas leñosas menores de 10 cm de DAP (Phillips \& Miller, 2002; Palacios-Ramos et al., 2018), y menos aún, aquellos que han analizado los tiempos de recomposición de los bosques generados a partir de perturbaciones antropogénicas (Echía et al., 2019).

Es clara la necesidad de incrementar investigaciones que brinden mayor información sobre las etapas de la recuperación florística y la diversidad arbórea asociada a diferentes edades de bosque. Por ello, en la presente pesquisa se propusieron los siguientes objetivos: (i) documentar la composición florística y la diversidad arbórea en diferentes edades de bosque, (ii) analizar las similitudes florísticas entre las diferentes edades de bosque, y (iii) precisar el tiempo de recuperación de los bosques húmedos premontanos del ámbito de estudio.

\section{Materiales y Métodos}

Ámbito de estudio

El ámbito de estudio está conformado por el bosque premontano, entre 1000 - $1500 \mathrm{msnm}$, en el valle de Chanchamayo, distrito de San Ramón, Dp. de Junín, Perú; allí se sitúan las áreas de bosque húmedo tropical de diferentes edades en las que se establecieron las unidades de muestra. El área de estudio y la clasificación ecológica por zonas de vida de Holdridge se observan en la Figura 1. El área central de muestreo se sitúa en el Instituto Regional de Desarrollo (IRD), Fundo La Génova, de la Universidad Nacional Agraria La Molina, el cual posee zonas de bosque intacto en sus partes más interiores, y un mosaico de bosques de diferentes edades en las partes más externas, hacia el río Chanchamayo. Tiene una extensión de 300 ha, de las cuales, aproximadamente, el 40\% están cubiertas por bosque húmedo premontano tropical, y el resto por cultivos tropicales, principalmente cítricos y piña. 

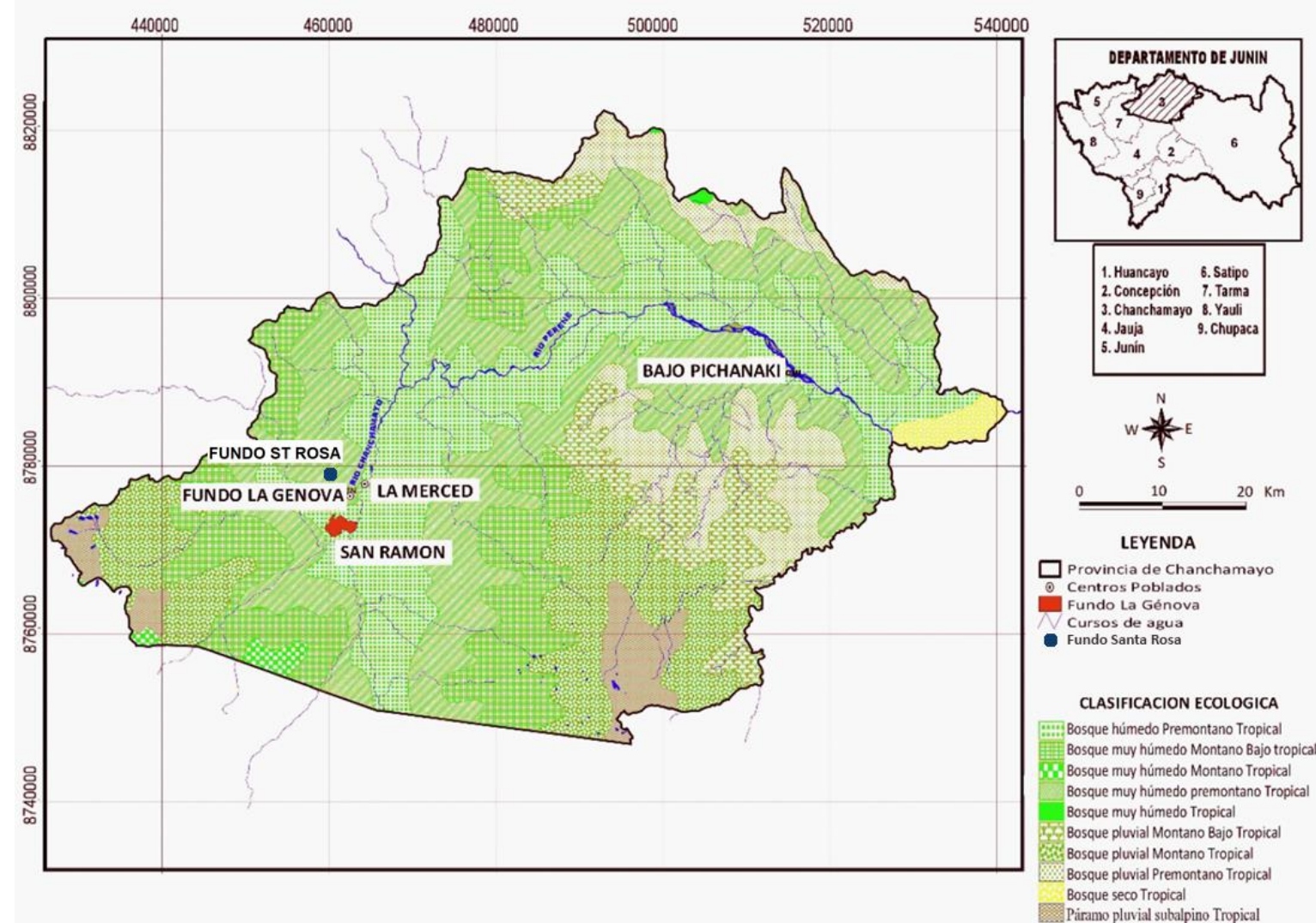

Fuente: Elaboración propia.

Figura 1. Estratificación del ámbito de estudio basada en el sistema de clasificación ecológica por zonas de vida de Holdridge.

Levantamiento de unidades de muestra en bosques de diferentes edades

Las unidades de muestra empleadas son los llamados Transectos Gentry (TG), conformados por una faja de evaluación de $2 \times 500 \mathrm{~m}$ a lo largo de la cual se registraron y colectaron todas las plantas $\geq 2.5 \mathrm{~cm}$ de DAP. Los TG fueron aleatoriamente establecidos en áreas de bosques húmedos de diferentes edades al interior del Fundo Santa Rosa y el IRD Fundo La Génova UNALM. La fuente de información principal sobre la edad de las áreas de bosque estudiadas fue la referencia de trabajadores de los fundos, varios de los cuales han vivido en el interior de éste desde su niñez. Se consideró como bosques maduros aquellas áreas que no han sido sufrido intervención humana en los últimos 50 años; y su estado de madurez se reflejó también en su ensamblaje de flora. Para las áreas de bosques de diferentes edades a lo largo de la sucesión vegetal se consideraron solamente aquellas resultantes de la intervención antropogénica sin quema (sucesión vegetal en bosques generados a partir de quemas puede ser consultada en Echía et al., 2019). Todas las plantas incluidas fueron colectadas, apropiadamente preservadas y acondicionadas para ser depositadas en el Herbario e identificadas por los taxónomos. Se levantaron al menos tres $\mathrm{TG}$ para cada edad aproximada de los bosques del ámbito, con un total de 16 TG. Los códigos de los TG levantados, con indicación de su posicionamiento y correspondientes edades, se muestran en la Tabla 1. Los especímenes botánicos colectados fueron depositados en el Herbario de la Facultad de Ciencias Forestales de la UNALM (colección de plantas leñosas del Herbario MOL), donde fueron determinados botánicamente por $\mathrm{C}$. Reynel y R. Fernandez-Hilario.

$\underline{\text { Análisis efectuados }}$

\section{DIVERSIDAD ALFA}

Se establecieron clases diamétricas de $5 \mathrm{~cm}$ para analizar el número de especies (Diversidad Alfa) que se registra en cada clase. Asimismo, para el caso de las clases altimétricas se establecieron clases de $5 \mathrm{~m}$ (Tabla 2). Los transectos fueron analizados en su conjunto para cada una de las edades estudiadas.

RECOMPOSICIÓN DE LA DIVERSIDAD ALFA (D $\alpha)$ EN BOSQUES DE DIFERENTES EDADES

En la comparación de la Diversidad Alfa (número de especies por unidad de área) entre unidades de muestra de diferentes edades, se tomaron en cuenta los valores absolutos de diversidad en cada caso, y también los valores de ésta obtenidos con el índice Alfa de Fisher, que corrige las diferencias ocasionadas por 
existir distintos números de individuos en cada Transecto Gentry (Fisher et al., 1943). En los estudios de diversidad arbórea, este índice muestra una alta estabilidad con respecto al tamaño de la muestra, ya que depende del número de individuos muestreados (Rosenzweig, 1995; Condit et al., 1996). Asimismo, este índice toma en cuenta el efecto positivo que tiene la abundancia sobre la diversidad, permitiéndole estimar la diversidad de áreas geográficas extensas usando muestras provenientes de áreas reducidas y comparar unidades de muestreo (Condit et al., 1996; Berry et al., 2002). El cálculo del índice alfa de Fisher se realizó mediante la Fórmula 1.

$$
\mathrm{S}=\alpha \ln [1+\mathrm{N} / \alpha]
$$

Fórmula 1. Cálculo para el índice alfa de Fisher.

Donde:

$\alpha=$ índice alfa de Fisher;

$\mathrm{S}=$ número de especies en la muestra;

$\mathrm{N}=$ número de individuos en la muestra.

El índice alfa de Fisher fue calculado con el programa PAST, versión 1.91 (Hammer et al., 2001).

COMPARACIÓN DE LA COMPOSICIÓN FLORÍSTICA EN BOSQUES DE DIFERENTES EDADES

La comparación se efectuó mediante el análisis de similitud con el uso del Índice de Dice (Dice, 1945) y un Análisis de Agrupamiento (Cluster Analysis). Para ambos casos se empleó el programa PAST versión 1.91 (Hammer et al., 2001).

\section{Resultados}

Recomposición de la Diversidad Alfa (D $\alpha)$

En lo que respecta a la recomposición de la $\mathrm{D} \alpha$ en bosques de diferentes edades (Tabla 3 ), se aprecia que los valores de este parámetro son muy modestos entre las edades de $<10$ años, haciéndose considerablemente mayores desde el rango de edad 20 años en adelante. Cuando se toma en consideración la cantidad de individuos registrados empleando el índice de diversidad Alfa de Fisher, se observa también que es a partir de 40 años en adelante que se produce una recuperación sustancial de la diversidad de especies arbóreas presentes.

La observación de los datos de los TG levantados muestra que, en todos los casos, las tres primeras clases diamétricas, $2.5-14.99 \mathrm{~cm}$ de diámetro, registran las abundancias más altas (Tabla 4). Los valores hallados sugieren que, a nivel de especies e individuos, existe una gran diferencia entre los bosques pioneros (menores a 10 años) y los bosques a partir de 20 años de edad. Asimismo, en la Figura 2 se puede apreciar (a excepción del bosque de $<10$ años) que las curvas reflejan una distribución de "J invertida" donde la mayor cantidad de individuos se hallan en las clases diamétricas menores. Esto indica una tendencia clara hacia la reposición del bosque. Con respecto a los resultados obtenidos para las clases altimétricas (Tabla 5 ), podemos apreciar que tanto la mayor cantidad de especies como individuos se hallan en la segunda clase $(5-9.99 \mathrm{~m})$, y si bien se esperaría una tendencia de "curva normal" en las clases altimétricas (Figura 3) esta tiende hacia la izquierda, agrupando las mayores cantidades de individuos en las tres primeras clases.

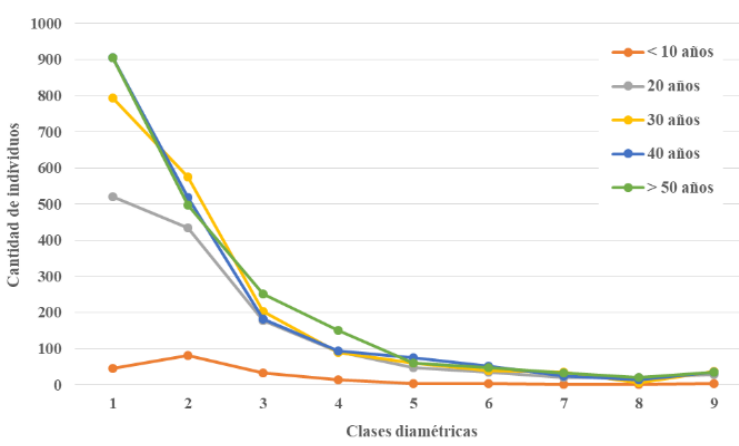

Figura 2. Clases diamétricas en las cinco edades de bosque evaluadas.

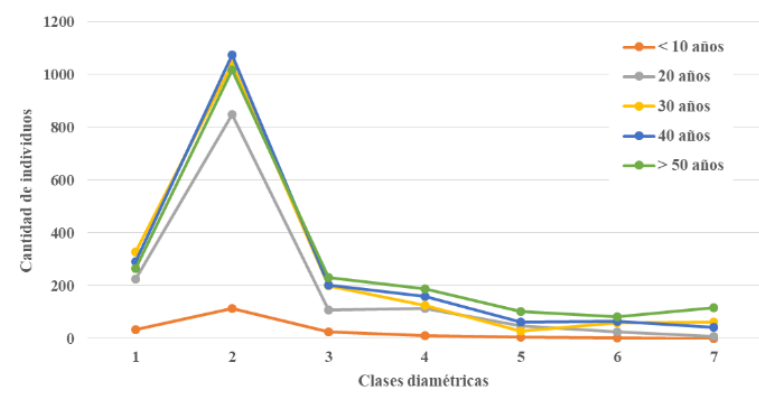

Figura 3. Clases altimétricas en las cinco clases de bosque evaluadas.

En cuanto a la composición por familias a lo largo de diferentes edades (Tabla 6), se perciben algunas tendencias de interés. Recién a partir de los 40 años, la familia Piperaceae deja de ser un componente marcadamente abundante en el bosque. Del mismo modo, a partir de los 20 años la familia Moraceae comienza a presentar una mayor cantidad de individuos, hasta llegar a ser la más importante en los bosques maduros ( $>50$ años). Con respecto a la diversidad, en todas las edades de bosque las familias Fabaceae y Lauraceae, de modo bastante estable, presentan las mayores cantidades de especies, aunque no necesariamente las especies son las mismas en todas las edades. Observamos también que en los bosques maduros ( $>50$ años), aparecen familias adicionales que no se habían registrado en edades anteriores (Connaraceae, Nyctagynaceae), y Annonaceae emerge como una de las familias más diversas.

ÍNDICE ALFA DE FISHER

Si bien la mayor cantidad de individuos y especies es registrada en las edades de bosque más avanzadas 
(40 y > 50 años), éstas no necesariamente representan los mayores valores de Alpha de Fisher. El máximo valor es registrado para bosques de 40 años. Es importante resaltar que los bosques de $<10$ años presentan una alta variabilidad Alpha de Fisher (5.78-14.66) y al menos dos de los transectos correspondientes muestran la menor diversidad de toda la evaluación realizada (Tabla 3).

RECOMPOSICIÓN DEL ENSAMBLAJE DE ESPECIES (COMPOSICIÓN FLORÍSTICA)

El análisis de similitud (índice de Dice; Tabla 7) revela que la composición florística de las edades tempranas (< 10 años) es poco compatible con la de bosques mayores de 20 años, con una similitud menor al $24 \%$. La similitud de los ensamblajes de especies se hace notoria en bosques a partir de 20 años en adelante, y muy notoria en bosques de 30 años en adelante, edad a partir de la cual $69-74 \%$ de las especies del bosque son las mismas que en edades más avanzadas. Bosques entre 30 y 40 años de edad son bastante similares entre sí en ese sentido; y los bosques mayores de 50 años tienen un ensamblaje de especies ligeramente diferenciado de ellos.

El análisis de agrupamiento (Figura 4) muestra que el bosque de $<10$ años forma un grupo solitario, con solamente $20 \%$ de similitud con el resto de edades. Por otra parte, los bosques de 30, 40 y > 50 años presentan una similitud aproximada de $70 \%$. Asimismo, las dos edades con mayor similitud (cerca del 70\%) son los bosques de 30 y 40 años. Esto sugiere que, en una primera etapa, cuando el bosque es pionero, existen muy pocas especies compartidas con los bosques maduros, y recién a partir de los 20 años de edad empiezan a proliferar aquellas especies que caracterizan los bosques maduros.

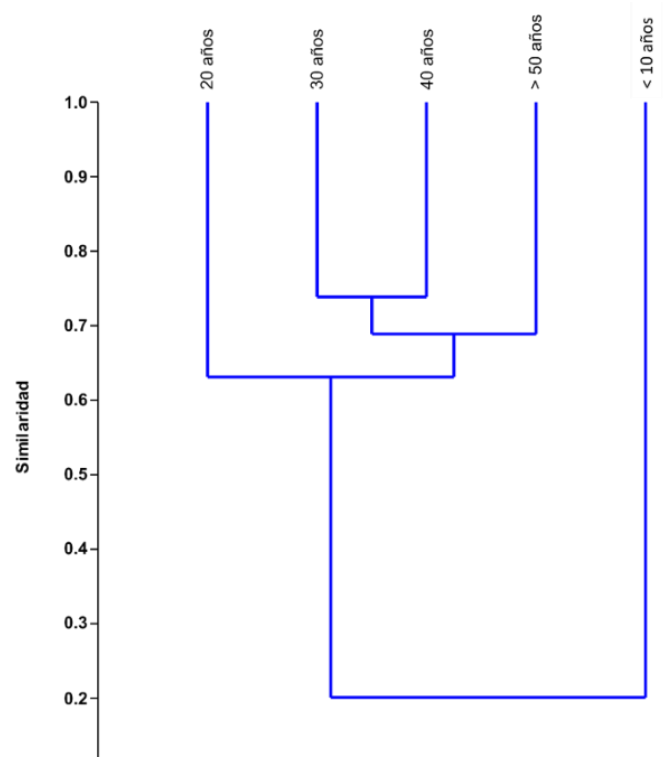

Figura 4. Dendrograma de similitud utilizando el Índice de Dice para las edades de bosque evaluadas.
Finalmente, observamos que pocas de las especies compartidas entre diferentes edades presentan gran abundancia (Tabla 8). La mayoría de especies compartidas presentan pocos individuos, siendo el caso extremo las especies en común entre los bosques de $<10$ años y > 50 años, donde sólo una presenta más de 10 individuos en ambas edades. A medida que se incrementa la edad de los bosques, existen menos especies compartidas con un alto número de individuos. Se llega a compartir siete especies con más de 50 individuos en los bosques de 40 años y $>50$ años. A partir de los 20 años, la especie Trophis caucana es un componente abundante en común con los bosques maduros (> 50 años).

\section{Discusión}

Los bosques húmedos premontanos de la Amazonía son una de las formaciones naturales con mayor impacto y alteración antropogénica en territorio peruano. La productividad en los bosques de montaña es menor que en tierras bajas, en consecuencia, las tasas de recuperación son relativamente más lentas, en un ambiente marcado por la heterogeneidad, en donde factores locales como los deslizamientos son comunes, y donde interactúan especies con historias evolutivas divergentes (Crausbay \& Martin, 2016). Un factor influyente en la recomposición de los bosques andinos tropicales es el efecto de borde de la vegetación circundante (Bueno \& Llambi, 2015). Se ha postulado que un área disturbada incrustada en una matriz de bosque circundante con una estructura y composición intactas tiene significativamente mejores posibilidades de recuperación que otra rodeada de bosques altamente degradados (Chadzon, 2003). Para comprender el proceso de recuperación de estos bosques, es importante aclarar el tiempo que ellos toman en recomponerse, desde el punto de vista de su diversidad y también de los ensamblajes de especies que los caracterizan. Sobre la base de dicha información sería posible, por ejemplo, encontrar alternativas técnicas para acortar dichos tiempos.

Los datos obtenidos muestran que, para bosques secundarios que no han sido sometidos a quemas, los estadios tempranos de la vegetación, también conocidos como pioneros (hasta 10 años de edad), son muy diferenciados en la abundancia y diversidad de especies contra los correspondientes bosques maduros, conteniendo una diversidad considerablemente menor. A diferencia de los bosques de Amazonía baja, en donde a los 20 años hay una recuperación del 80\% de la riqueza de especies (Rozendaal et al., 2019), los momentos más importantes en la recomposición de estos bosques se muestran sobre todo a partir de 30 años de edad, tiempo en el cual se alcanza una similitud cercana a $70 \%$ con los bosques maduros. Una interrogante abierta a futura investigación es qué propiedades son alcanzadas a partir de estas edades, que permiten esa rápida recuperación de la diversidad y el 
ensamblaje de especies característico de formaciones de bosque maduro. La tasa de recuperación podría estar limitada por la fertilidad del suelo y el tipo de suelo (Chadzon, 2003); en ese sentido, especulativamente, podría suponerse que propiedades importantes relacionadas a la fertilidad y capacidad de carga del suelo, así como el aumento de interventores bióticos como dispersadores de semillas, y el microclima producido en el bosque, podrían mostrar cambios sustanciales a partir de esos momentos.

De otro lado, en el caso del bosque húmedo premontano estudiado, la presencia de algunos taxones podría interpretarse como indicadora de la edad y estado de recuperación del bosque; Piperaceae es una familia característicamente presente en las edades tempranas, perdurando como abundante hasta los 3040 años, representada mayoritariamente por la especie Piper aduncum; Moraceae, representada por la especie Trophis caucana, se percibe como una familia indicadora de la condición madura del bosque en el bosque estudiado. Lo mencionado coincide con las observaciones de Gentry \& Ortiz (1993). Si comparamos estos datos con los obtenidos en bosques que se regeneran luego de quemas (Echía et al., 2019), observamos que en estos últimos la recuperación es aparentemente más lenta. Considerando el rango de edad entre 25-30 años, en los últimos el 57\% de la diversidad se recupera en ese lapso, vs. 69-74\% en los bosques que no han estado sometidos a quemas, analizados en el presente estudio.

\section{Conclusiones}

La composición florística en diferentes edades en los bosques húmedo premontanos estudiados es reconocible; adicionalmente la presencia y abundancia de algunos taxones puede interpretarse como indicadora de la edad y estado de recuperación del bosque; por ejemplo bosques secundarios tempranos, de hasta 20 años de edad, son caracterizados por la abundancia de la familia Piperaceae, teniendo como especie más abundante a Piper aduncum, mientras que bosques maduros, a partir de 30 años de edad, lo son por la familia Moraceae, con la especie Trophis caucana.

Desde el punto de vista de su diversidad y su ensamblaje de flora, los bosques tempranos, menores a 20 años de edad, son significativamente menos diversos, y diferenciados en su composición florística con bosques mayores de 30 años (menos del 24\% de similitud); a partir de esta edad, la diversidad de especies se eleva notoriamente.

En los bosques húmedos premontanos del ámbito de estudio, los momentos más importantes en la recomposición de la diversidad y el ensamblaje de especies se producen a partir de 20 años de edad, y sobre todo a partir de 30 años de edad, tiempo en el cual se alcanza una similitud cercana a $70 \%$ con la composición de la flora de bosques maduros.

\section{Agradecimientos}

Los autores agradecen a todo el personal del Círculo de Investigación DINAFOR por su colaboración durante las fases de campo y gabinete. Esta investigación ha sido financiada por Cienciactiva, CONCYTEC.

\section{Literatura citada}

Aguilar M. \& Reynel C. 2011. Dinámica forestal y regeneración en un bosque montano nublado de la selva central del Perú. Herbario de la Facultad de Ciencias Forestales, Universidad Nacional Agraria La Molina. Lima, Perú. URL: http://www.aprodes.org/pdf/dinamica_bosque_montano. pdf.

Almeyda A. 2004. Diversidad y composición de la flora arbórea en un área de bosque secundario tardío: Fundo Génova-UNALM, valle de Chanchamayo, 1000-1500 msnm. En: Antón D. \& Reynel C. (Eds.) Relictos de bosques de excepcional diversidad en los andes centrales del Perú. 263-302. Herbario de la Facultad de Ciencias Forestales, Universidad Nacional Agraria La Molina. Lima, Perú. URL: http://www.infobosques.com/descargas/biblioteca/446.p df.

Antón D. \& Reynel C. (Eds.). 2004. Relictos de bosques de excepcional diversidad en los andes centrales del Perú. Herbario de la Facultad de Ciencias Forestales, Universidad Nacional Agraria La Molina. Lima, Perú. URL:

http://www.infobosques.com/descargas/biblioteca/446.p df.

Arroyo F. 2014. A new species of Magnolia (Magnoliaceae) from central Peru. Phytotaxa, 167(2): 220-222. DOI: 10.11646/Phytotaxa.167.2.14.

Berg C.C. \& Ulloa C. 2014. Two new species of Coussapoa (Urticaceae, Cecropieae). Novon: A Journal for Botanical Nomenclature, 23(1): 14-17. DOI: 10.3417/2013002.

Berry P.E., Guariguata M.R. \& Kattan G.H. 2002. Diversidad y endemismo en los bosques neotropicales de bajura. En: Guariguata M.R. \& Kattan G.H. (Eds.) Ecología y conservación de bosques neotropicales, 83-96. Editorial Tecnológica. Cartago, Costa Rica.

Bueno A. \& Llambí L. 2015. Facilitation and edge effects influence vegetation regeneration in old-fields at the tropical Andean forest line. Applied Vegetation Science, 18(4): 613-623. DOI: 10.1111/avsc. 12186.

Buttgenbach H., Vargas C. \& Reynel C. 2012. Dinámica forestal en un bosque premontano del valle de Chanchamayo (Dp. de Junín, 1200 msnm). Herbario de la Facultad de Ciencias Forestales, Universidad Nacional Agraria La Molina. Lima, Perú. URL: http://www.aprodes.org/pdf/dinamica_bosque_premonta no.pdf.

Cáceres P. \& Reynel C. 2010. Los árboles de Ficus (“Ojé”) del valle de Chanchamayo, Dpto. Junín, Perú (800-2500 msnm). Asociación Peruana para la Promoción del Desarrollo Sostenible (APRODES). Lima, Perú. URL: http://www.aprodes.org/pdf/arboles_ficus.pdf.

Chadzon R. 2003. Tropical forest recovery: legacies of human impact and natural disturbances. Perspectives in Plant Ecology, Evolution and Systematics, 6(1-2): 51-71. DOI: 10.1078/1433-8319-00042. 
Condit R., Hubbell S.P., Lafrankie J.V., Sukumar R., Manokaran N., Foster R. \& Ashton P. 1996. SpeciesArea and Species-Individual relationships for tropical trees: A comparison of three 50 ha plots. Journal of Ecology, 84(4): 549-562. DOI: 10.2307/2261477. https://www.jstor.org/stable/2261477.

Crausbay Sh. \& Martin P. 2016. Natural disturbance, vegetation patterns and ecological dynamics in tropical montane forests. Journal of Tropical Ecology, 32(5): 384 403. DOI: $10.1017 / \mathrm{S} 0266467416000328$.

Dice L.R. 1945. Measures of the amount of ecologic association between species. Ecology, 26(3): 297-302. DOI: https://www.jstor.org/stable/1932409.

Echía E., Reynel C. \& Manta I. 2019. La flora leñosa establecida luego de las quemas en el valle de Chanchamayo - Selva central del Perú. Revista Forestal del Perú, 34(1): 83-101. DOI: 10.21704/rfp.v34i1.1287.

Fisher R.A., Corbet A.S. \& Williams C.B. 1943. The relation between the number of species and the number of individuals in a random sample of an animal population. The Journal of Animal Ecology, 12(1): 42-58. DOI: 10.2307/1411. https://www.jstor.org/stable/1411.

Gentry A. \& Ortiz R. 1993. Patrones de composición florística en la Amazonia peruana. En: Kalliola R., Puhakka M. \& Danjoy W. (Eds.). Amazonia peruana, vegetación húmeda tropical en el llano subandino. 155166. PAUT y ONERN. Lima, Perú. URL: http://www.mobot.org/MOBOT/Research/curators/pdf/ Gentry-Ortiz-1993.pdf.

Giacomotti J. \& Reynel C. 2018. Mortalidad y reclutamiento de árboles en un bosque secundario tardío del valle de Chanchamayo, Perú. Revista Forestal del Perú, 33(1): 42 51. DOI: $10.21704 / \mathrm{rfp} . v 33 i 1.1154$.

Hammer Ø., Harper D.A.T. \& Ryan P.D. 2001. PAST: Paleontological Statistics Software Package for Education and Data Analysis. Paleontología Electrónica, 4(1): $\quad 1-9 . \quad \mathrm{https} / / /$ palaeoelectronica.org/2001_1/past/past.pdf.

Honorio E. \& Reynel C. 2003. Vacíos en la colección de la flora de los Bosques Húmedos del Perú. Herbario de la Facultad de Ciencias Forestales, Universidad Nacional Agraria La Molina. Lima, Perú. URL: http://siar.minam.gob.pe/puno/sites/default/files/archivo s/public/docs/1225.pdf.

Marcelo J. \& Reynel. C. 2014. Patrones de diversidad y composición florística de parcelas de evaluación permanente en la selva central del Perú. Rodriguesia, 65(1): 35-47. DOI: 10.1590/S2175-78602014000100003.

Monteagudo A. \& Huamán M. 2010. Catálogo de los árboles y afines de la Selva Central del Perú. Arnaldoa, 17(2): 203-242.

URL: http://www.rainfor.org/upload/publicationstore/itm_26/Monteagudo.pdf.

Palacios S. \& Reynel C. 2011. Una formación vegetal subxerófila en el valle de Chanchamayo, Dp. Junín. Herbario de la Facultad de Ciencias Forestales, Universidad Nacional Agraria La Molina. Lima, Perú. URL:

http://www.aprodes.org/pdf/formacion_vegetal.pdf.
Palacios-Ramos S., Montenegro R., Linares-Palomino L. \& Reynel C. 2018. Forest dynamics of a sub-xeophylous vegetation formation in central Peru - Chanchamayo valley, Peru. Revista Árvore, 42(6): e420603. DOI: 10.1590/1806-90882018000600003.

Phillips O., Baker T., Feldpausch T. \& Brienen R. 2009. RAINFOR Manual de campo para la remedición y establecimiento de parcelas permanentes. URL: https://www.forestplots.net/upload/es/recursos/RAINFO R_field_manual_ES.pdf.

Phillips O. \& Miller J. 2002. Global patterns of plant diversity: Alwyn H. Gentry's forest transect data set. MSB (Monographs in Systematic Botany), 89: 1-319. Missouri Botanical Garden Press.

Pipoly III J.J. 1996. New species of Geissanthus (Myrsinaceae) from the Hylaea/Andean interface of Ecuador and Peru. SIDA, Contributions of botany, 17(2): 459-470. https://www.jstor.org/stable/41967229.

Reynel C. 2012. Flora y fauna del bosque montano nublado Puyu Sacha, valle de Chanchamayo, Dp. Junín (18003200 msnm). Asociación Peruana para la Promoción del Desarrollo Sostenible (APRODES). Lima, Perú. URL: https://www.puyusacha.org/archivos/publicaciones/pdf_ 49_p7.pdf.

Reynel C. \& Antón D. 2004. Diversidad y composición de la flora arbórea en un área de cumbre de colinas en bosque premontano: Fundo Génova-UNALM, valle de Chanchamayo, 1000-1500 msnm. En: Antón, D. \& Reynel, C. (Ed.). Relictos de bosques de excepcional diversidad en los andes centrales del Perú. 143-186. Herbario de la Facultad de Ciencias Forestales, Universidad Nacional Agraria La Molina. Lima, Perú. URL:

http://www.infobosques.com/descargas/biblioteca/446.p df.

Reynel C. \& León J. 1989. Especies forestales comunes de los bosques secundarios de Chanchamayo, Perú. ICRD /UNALM. Lima, Perú.

Rosenzweig M.L. 1995. Species diversity in space and time. Universidad de Cambridge, Cambridge, Reino Unido. DOI: $10.1017 / \mathrm{CBO} 9780511623387$.

Rozendaal D.M., Bongers F., Mitchell Aide T., AlvarezDávila E., Ascarrunz N., Balvanera P., et al. 2019. Biodiversity recovery of Neotropical secondary forests. Science Advances, 5(3): eaau3114. DOI: 10.1126/sciadv.aau3114.

Vásquez R., Rojas R., Monteagudo A., Van der Werff H. \& Ortiz R. 2005. Flora vascular de la selva central del Perú: una aproximación de la composición florística de tres Áreas Naturales Protegidas. Arnaldoa, 12(1-2): 112-125. URL: http://www.infobosques.com/descargas/biblioteca/371.p df. 
Tabla 1. Ubicación de los Transectos Gentry de 0.1 ha establecidos en diferentes edades aproximadas de bosque.

\begin{tabular}{|c|c|c|c|c|c|c|}
\hline \multirow{2}{*}{$\begin{array}{l}\text { Edades } \\
\text { aproximadas }\end{array}$} & \multirow[t]{2}{*}{ Transectos } & \multicolumn{3}{|c|}{ Coordenadas } & \multirow{2}{*}{$\begin{array}{l}\text { Altitud } \\
\text { (msnm) }\end{array}$} & \multirow[t]{2}{*}{ Ubicación } \\
\hline & & Zona & Este & Sur & & \\
\hline \multirow[t]{4}{*}{$<10$ años } & T1 & $18 \mathrm{~L}$ & 461463 & 8776420 & 1150 & Santa Rosa \\
\hline & $\mathrm{T} 2$ & $18 \mathrm{~L}$ & 461529 & 8776525 & 1150 & Santa Rosa \\
\hline & $\mathrm{T} 3$ & $18 \mathrm{~L}$ & 461419 & 8776802 & 1150 & Santa Rosa \\
\hline & $\mathrm{T} 4$ & $18 \mathrm{~L}$ & 461479 & 8776781 & 1150 & Santa Rosa \\
\hline \multirow[t]{3}{*}{20 años } & T5 & $18 \mathrm{~L}$ & 460753 & 8773466 & 1190 & IRD La Génova \\
\hline & T6 & $18 \mathrm{~L}$ & 460870 & 8773519 & 1170 & IRD La Génova \\
\hline & $\mathrm{T} 7$ & $18 \mathrm{~L}$ & 460986 & 8773493 & 1130 & IRD La Génova \\
\hline \multirow[t]{3}{*}{30 años } & $\mathrm{T} 8$ & $18 \mathrm{~L}$ & 460284 & 8773083 & 1280 & IRD La Génova \\
\hline & T9 & $18 \mathrm{~L}$ & 460367 & 8773166 & 1240 & IRD La Génova \\
\hline & T10 & $18 \mathrm{~L}$ & 460492 & 8773232 & 1230 & IRD La Génova \\
\hline \multirow[t]{3}{*}{40 años } & $\mathrm{T} 11$ & $18 \mathrm{~L}$ & 460437 & 8773458 & 1260 & IRD La Génova \\
\hline & $\mathrm{T} 12$ & $18 \mathrm{~L}$ & 460465 & 8773384 & 1230 & IRD La Génova \\
\hline & T13 & $18 \mathrm{~L}$ & 460613 & 8773484 & 1290 & IRD La Génova \\
\hline \multirow[t]{3}{*}{$>50$ años } & T14 & $18 \mathrm{~L}$ & 460823 & 8772416 & 1116 & IRD La Génova \\
\hline & T15 & $18 \mathrm{~L}$ & 460841 & 8772586 & 1152 & IRD La Génova \\
\hline & T16 & $18 \mathrm{~L}$ & 460881 & 8772617 & 1136 & IRD La Génova \\
\hline
\end{tabular}

Tabla 2. Clases diamétricas y altimétricas establecidas para el análisis de la Diversidad Alfa en los Transectos Gentry.

\begin{tabular}{lll}
\hline Clases & & Intervalos $(\mathbf{c m})$ \\
\hline Diamétricas & 1 & $2.5-4.99$ \\
& 2 & $5-9.99$ \\
& 3 & $10-14.99$ \\
& 4 & $15-19.99$ \\
& 5 & $20-24.99$ \\
& 6 & $25-29.99$ \\
& 7 & $30-34.99$ \\
Altimétricas & 8 & $35-39.99$ \\
& 9 & $>40$ \\
& 1 & $1-4.99$ \\
& 2 & $5-9.99$ \\
& 3 & $10-14.99$ \\
& 4 & $15-19.99$ \\
& 5 & $20-24.99$ \\
& 6 & $25-29.99$ \\
& 7 & $>30$ \\
\hline
\end{tabular}

Tabla 3. Valores de especies, familias e individuos en las diferentes edades de bosque evaluados. El Alfa de Fisher es el valor promedio hallado para los transectos de cada edad.

\begin{tabular}{|c|c|c|c|c|c|c|c|c|}
\hline \multirow{2}{*}{$\begin{array}{c}\begin{array}{c}\text { Edades } \\
\text { aproximadas }\end{array} \\
<10 \text { años }\end{array}$} & \multirow{2}{*}{$\begin{array}{l}\text { Transectos } \\
\text { T1 }\end{array}$} & \multicolumn{2}{|c|}{ Especies } & \multirow{2}{*}{$\begin{array}{c}\text { Familias } \\
19\end{array}$} & \multicolumn{2}{|c|}{ Individuos } & \multicolumn{2}{|c|}{ Alpha de Fisher } \\
\hline & & 11 & 48 & & 33 & 188 & 5.78 & 10.2 \\
\hline & $\mathrm{T} 2$ & 16 & & & 29 & & 14.66 & \\
\hline & T3 & 18 & & & 62 & & 8.51 & \\
\hline & $\mathrm{T} 4$ & 22 & & & 64 & & 11.85 & \\
\hline \multirow[t]{3}{*}{20 años } & T5 & 58 & 95 & 32 & 491 & 1378 & 17.1 & 17.9 \\
\hline & T6 & 55 & & & 471 & & 16.14 & \\
\hline & $\mathrm{T} 7$ & 63 & & & 416 & & 20.64 & \\
\hline \multirow[t]{3}{*}{30 años } & $\mathrm{T} 8$ & 56 & 87 & 28 & 601 & 1841 & 15.1 & 15.4 \\
\hline & T9 & 55 & & & 600 & & 14.74 & \\
\hline & $\mathrm{T} 10$ & 60 & & & 640 & & 16.21 & \\
\hline \multirow[t]{3}{*}{40 años } & $\mathrm{T} 11$ & 80 & 108 & 33 & 683 & 1897 & 23.51 & 18.7 \\
\hline & T12 & 63 & & & 578 & & 18 & \\
\hline & T13 & 56 & & & 636 & & 14.8 & \\
\hline \multirow[t]{3}{*}{ > 50 años } & $\mathrm{T} 14$ & 63 & 97 & 33 & 709 & 2002 & 16.7 & 18.4 \\
\hline & $\mathrm{T} 15$ & 75 & & & 684 & & 21.48 & \\
\hline & T16 & 61 & & & 609 & & 16.88 & \\
\hline
\end{tabular}


Tabla 4. Número de especies, e individuos (entre paréntesis) por clase diamétrica, en las edades de bosque evaluadas.

\begin{tabular}{cccccc}
\hline Clase diamétrica & $<\mathbf{1 0}$ años & 20 años & 30 años & 40 años & $>$ 50 años \\
\hline 1 & $24(45)$ & $63(520)$ & $68(793)$ & $82(905)$ & $71(906)$ \\
2 & $31(82)$ & $68(434)$ & $59(576)$ & $71(518)$ & $59(497)$ \\
3 & $14(32)$ & $39(178)$ & $46(204)$ & $48(182)$ & $39(252)$ \\
4 & $9(15)$ & $34(92)$ & $27(90)$ & $33(94)$ & $42(151)$ \\
5 & $3(3)$ & $19(47)$ & $21(60)$ & $30(74)$ & $26(61)$ \\
6 & $4(4)$ & $12(36)$ & $15(40)$ & $17(51)$ & $25(47)$ \\
7 & $2(2)$ & $10(21)$ & $16(34)$ & $12(25)$ & $20(33)$ \\
8 & $2(2)$ & $4(21)$ & $4(6)$ & $7(13)$ & $14(20)$ \\
9 & $3(3)$ & $5(29)$ & $11(38)$ & $15(35)$ & $10(35)$ \\
\hline
\end{tabular}

Tabla 5. Número de especies, e individuos (entre paréntesis), por clase altimétrica en las edades de bosque evaluados.

\begin{tabular}{ccclll}
\hline Clase de altura & $<\mathbf{1 0}$ años & 20 años & 30 años & 40 años & $>$ 50 años \\
\hline 1 & $16(32)$ & $50(225)$ & $51(329)$ & $55(292)$ & $46(264)$ \\
2 & $35(114)$ & $77(849)$ & $72(1040)$ & $85(1075)$ & $71(1017)$ \\
3 & $13(25)$ & $37(107)$ & $51(198)$ & $48(203)$ & $39(232)$ \\
4 & $7(11)$ & $29(114)$ & $33(124)$ & $47(158)$ & $37(189)$ \\
5 & $4(5)$ & $13(49)$ & $13(29)$ & $25(61)$ & $33(103)$ \\
6 & $1(1)$ & $5(26)$ & $15(60)$ & $23(66)$ & $30(82)$ \\
7 & $0(0)$ & $1(8)$ & $12(61)$ & $13(42)$ & $29(115)$ \\
\hline
\end{tabular}

Tabla 6. Las diez familias más diversas y abundantes para cada edad de bosque evaluado.

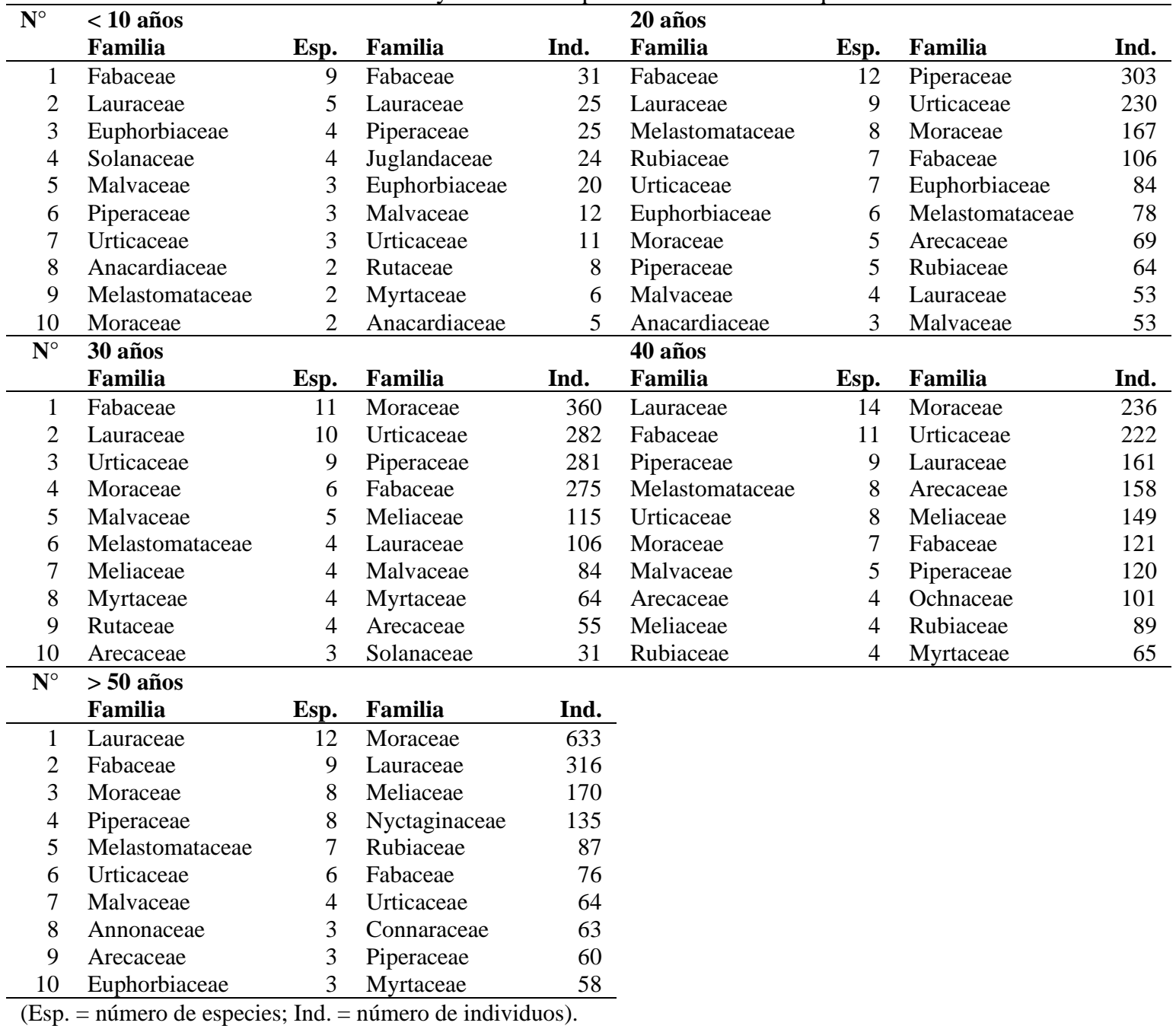


Tabla 7. Similitud entre grupos de edad en función al Índice de Dice.

\begin{tabular}{lccccc}
\hline Edades aproximadas & $<\mathbf{1 0}$ años & $\mathbf{2 0}$ años & 30 años & 40 años & $>$ 50 años \\
\hline$<10$ años & 48 & 0.238 & 0.222 & 0.192 & 0.152 \\
20 años & 17 & 95 & 0.659 & 0.640 & 0.594 \\
30 años & 15 & 60 & 87 & 0.738 & 0.685 \\
40 años & 15 & 65 & 72 & 108 & 0.693 \\
$>50$ años & 11 & 57 & 63 & 71 & 97 \\
\hline
\end{tabular}

A lo largo de la diagonal (de izquierda a derecha y de arriba a abajo) se encuentra el número de especies por edades de bosque. Por debajo de la diagonal el número de especies en común entre las edades de bosque, y por arriba el índice de Dice. Los mínimos y máximos valores registrados están resaltados en naranja y verde, respectivamente.

Tabla 8. Especies en común entre el bosque maduro (> 50 años) y las demás edades de bosque evaluadas y en función de una mínima cantidad de individuos registrados.

\begin{tabular}{|c|c|c|c|c|c|}
\hline \multirow[b]{2}{*}{$\begin{array}{l}\text { Edad } \\
\text { aproximada } \\
\text { de bosque }\end{array}$} & \multicolumn{5}{|c|}{$\begin{array}{c}>50 \text { años } \\
\text { Especies en común }\end{array}$} \\
\hline & $\begin{array}{l}\text { En } \\
\text { total }\end{array}$ & $\begin{array}{c}\text { con }>10 \\
\text { individuos } \\
\text { en ambas } \\
\text { edades }\end{array}$ & $\begin{array}{c}\text { con }>20 \\
\text { individuos } \\
\text { en ambas } \\
\text { edades }\end{array}$ & $\begin{array}{c}\text { con }>30 \\
\text { individuos } \\
\text { en ambas } \\
\text { edades }\end{array}$ & $\begin{array}{c}\text { con }>50 \text { individuos } \\
\text { en ambas edades }\end{array}$ \\
\hline$<10$ años & 11 & 1 & - & - & - \\
\hline 20 años & 57 & 10 & 3 & 2 & 1 (Trophis caucana) \\
\hline 30 años & 63 & 20 & 10 & 5 & $\begin{array}{l}3 \text { (Myrcia splendens; } \\
\text { Guarea guidonia; } \\
\text { Trophis caucana) }\end{array}$ \\
\hline 40 años & 71 & 23 & 13 & 8 & $\begin{array}{l}7 \text { (Prunus debilis; } \\
\text { Ruagea glabra; } \\
\text { Myrcia splendens; } \\
\text { Ocotea cernua; } \\
\text { Clarisia racemosa; } \\
\text { Guarea guidonia; } \\
\text { Trophis caucana) }\end{array}$ \\
\hline
\end{tabular}

${ }^{1}$ Herbario Forestal MOL, Facultad de Ciencias Forestales, Universidad Nacional Agraria La Molina. Av. La Molina s/n, La Molina, Lima, Perú.

${ }^{2}$ Servicio Forestal y de Fauna Silvestre (SERFOR). Av. Javier Prado Oeste N²442, Urb. Orrantia, Magdalena del Mar, Lima, Perú.

${ }^{3}$ Autor de correspondencia. reynel@lamolina.edu.pe.

${ }^{4}$ División de Ecología Vegetal - CORBIDI, Calle Santa Rita 105 Of. 2, Urb. Huertos de San Antonio Monterrico, Surco, Lima, Perú. 